\title{
EchoGéo
}

$10 \mid 2009$

La piraterie

\section{La lutte contre la piraterie au large de la Somalie}

De l'action nationale à l'action européenne (2008-2009)

Vice-amiral Gérard Valin

2 OpenEdition

Journals

Édition électronique

URL : https://journals.openedition.org/echogeo/11338

DOI : 10.4000/echogeo. 11338

ISSN : 1963-1197

Éditeur

Pôle de recherche pour l'organisation et la diffusion de l'information géographique (CNRS UMR 8586)

Référence électronique

Vice-amiral Gérard Valin, «La lutte contre la piraterie au large de la Somalie », EchoGéo [En ligne], 10 | 2009, mis en ligne le 24 novembre 2009, consulté le 16 septembre 2021. URL : http://

journals.openedition.org/echogeo/11338; DOI : https://doi.org/10.4000/echogeo.11338

Ce document a été généré automatiquement le 16 septembre 2021.

EchoGéo est mis à disposition selon les termes de la licence Creative Commons Attribution - Pas d'Utilisation Commerciale - Pas de Modification 4.0 International (CC BY-NC-ND) 


\section{La lutte contre la piraterie au large de la Somalie}

De l'action nationale à l'action européenne (2008-2009)

Vice-amiral Gérard Valin

1 Placé directement sous l'autorité du chef d'état-major des armées, le vice-amiral Gérard Valin, commandant la zone maritime de l'océan Indien (ALINDIEN de 2008 à 2009) assume le commandement des forces françaises déployées dans ce vaste espace placé au cœur de ce que le Livre Blanc qualifie « d'arc stratégique prioritaire pour la défense des intérêts de la France ». Cette zone, sujette à de nombreux foyers de crises ouvertes ou potentielles, va de l'Atlantique à l'océan Indien, jusqu'en Asie du Sud-Est «nouveau centre de gravité stratégique » et « principale zone de croissance du monde $»^{1}$.

2 Exerçant ses fonctions depuis le mois de février 2008, le vice-amiral Gérard Valin, avec son état-major interarmées et l'ensemble des forces placées sous ses ordres, a très rapidement été confronté à la montée exponentielle de la piraterie au large des côtes somaliennes. Les trois opérations de libération des otages des voiliers Le Ponant, Carré d'As IV et Tanit qu'il a conduites en avril et septembre 2008 et en avril 2009, mais aussi l'action au quotidien illustrent cette nouvelle approche de défense et de sécurité imposée par la mondialisation et fondée sur les deux capacités clés que sont l'anticipation et la réactivité.

3 L'action déterminée de la France dans la lutte contre la piraterie et la forte volonté du président de la République à mobiliser la communauté internationale contre ce fléau ont abouti à plusieurs résolutions du Conseil de sécurité des Nations-Unies et au lancement de l'opération Atalanta, première opération maritime de l'Union Européenne, au sein de laquelle opèrent désormais les unités de la marine française.

L'exemple de la lutte internationale contre la piraterie dans le golfe d'Aden et le rôle de pionnier de la France en la matière ont montré tout l'intérêt du prépositionnement permanent de moyens français au cœur de cette zone éminemment stratégique. 


\section{Importance stratégique de la zone de l'océan Indien}

5 L'une des données essentielles du théâtre est le flux stratégiquement sensible, à l'échelle mondiale, des approvisionnements en hydrocarbures. La France se doit de préserver ses intérêts économiques et commerciaux dans cette zone qui voit passer une part prépondérante du commerce, chaque année plus importante, entre l'Europe et l'Asie. La sécurité des routes maritimes de cet océan, notamment celles passant par les détroits de Bab El Mandeb, d'Ormuz et de Malacca, est de première importance pour notre pays. Les espaces maritimes constituant le réseau sanguin de la mondialisation, la croissance économique et la prospérité du monde en dépendent directement.

6 Au sein de cette zone complexe et instable, l'Asie du Sud-Est, qui rassemble des pays à forte croissance, connaît un développement particulièrement rapide. Des pays comme la Malaisie, Singapour et l'Indonésie font preuve d'un dynamisme économique stimulant, de nature à entraîner dans leur sillage les autres pays d'une région qui, trait d'union entre les deux géants démographiques de la planète, la Chine et l'Inde, devient progressivement le nouveau centre de gravité d'un monde en pleine mutation.

7 La France, pays riverain de l'océan Indien, a toute sa place dans cet espace émergeant. Il lui faut protéger ses citoyens, plus d'un million, ainsi que les territoires et les zones maritimes placés sous sa souveraineté : îles de la Réunion, Mayotte, îles Eparses, Terres australes et antarctique française (TAAF). Les zones économiques de ces territoires représentent plus de 2,5 millions de $\mathrm{km}^{2}$, soit sept fois la surface de la ZEE métropolitaine. Cette présence ancienne de la France lui a permis de tisser des liens historiques, culturels et de défense avec les pays de la région. Elle est actuellement liée par des accords de défense ou de coopération militaire avec une douzaine d'Etats de l'océan Indien.

8 La France doit par ailleurs remplir les obligations d'acteur international que lui confère son siège de membre permanent au Conseil de Sécurité de l'ONU : la déstabilisation d'une région entraîne de telles conséquences pour l'ensemble de la planète que les grandes puissances, s'appuyant sur des moyens de projection de puissance et de force ${ }^{2}$, mais aussi sur des capacités de forces spéciales, doivent être capables d'intervenir loin de leurs frontières pour gérer les crises et participer à la stabilité de la planète.

\section{L'explosion de la piraterie dans le golfe d'Aden}

9 Lieu de multiples activités illicites, du trafic d'armes ou de drogue aux exactions terroristes, cette zone est devenue le théâtre d'une recrudescence hors norme des actes de piraterie de nature à déstabiliser le commerce mondial. Désormais relativement marginale en mer de Chine et dans le détroit de Malacca, maintenant que les quatre Etats concernés - Singapour, Malaisie, Indonésie et Thaïlande - conjuguent leurs efforts dans des patrouilles efficaces, la piraterie a connu en 2008 un essor remarquable au large de la Somalie, en particulier dans le golfe d'Aden. Une zone que franchissent chaque année près de 20000 navires et par laquelle transitent $30 \%$ des approvisionnements européens en pétrole.

Douze attaques et tentatives d'actes de piraterie avaient été recensées en 2006 et 35 en 2007. En 2008, on atteint les chiffres hors normes d'environ 170 tentatives dont 45 avec succès. En 2009, à la fin du mois de juin, on dénombrait déjà 25 navires piratés pour 
133 tentatives. A l'heure actuelle, douze navires et plus de 160 a Q A12 otages sont toujours détenus par les pirates.

11 Ecumant la quasi-totalité du golfe d'Aden et une large zone maritime à l'est du littoral somalien, les pirates opèrent sur un espace toujours plus vaste, couvrant désormais une surface équivalente à quatre fois la superficie de la France. Ils sont particulièrement déterminés et bien organisés. Constitués pour la plupart en véritables groupes paramilitaires de plusieurs centaines de personnes, ils se répartissent entre équipes d'attaque en mer et miliciens chargés, au mouillage, de la détention des navires et des otages.

Photo 1 - Un skiff et son armement complet

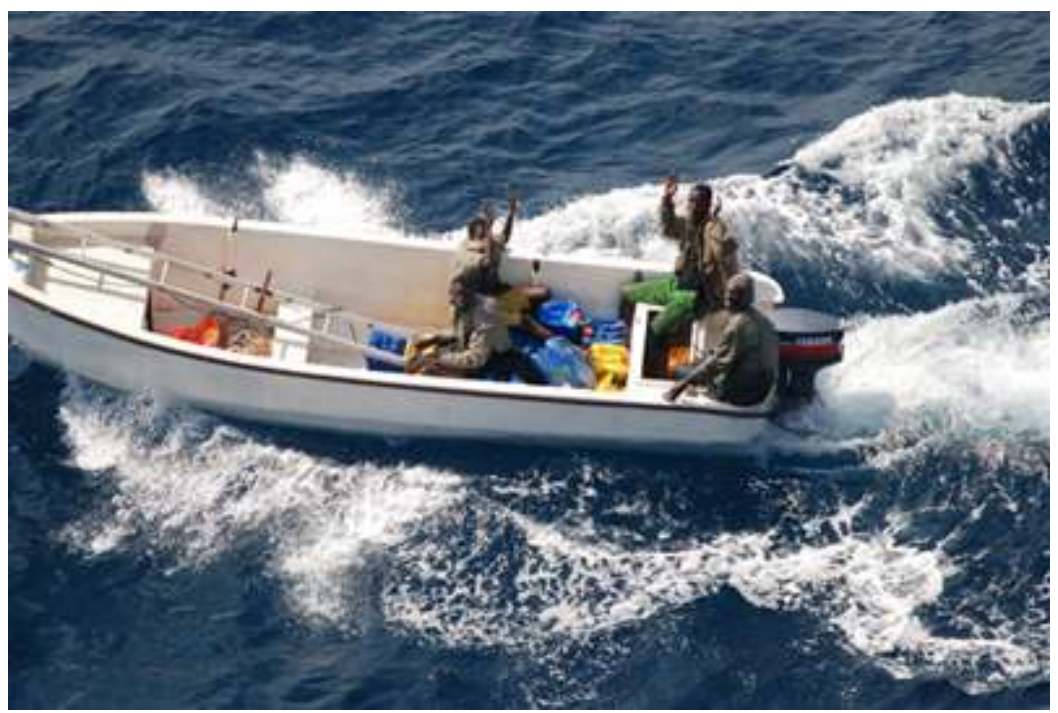

Cliché : auteur.

12 Les équipes d'assaut peuvent compter jusqu'à une dizaine de pirates, armés le plus souvent de fusils mitrailleurs et de lance-roquettes. Les pirates sont en mesure de conduire des attaques à grande distance des côtes somaliennes. Depuis 2007, de nombreux assauts se sontdéroulés à plus de 200 nautiques des côtes. Le cas emblématique du super tanker saoudien Sirius Star a même été rapporté à plus de 450 milles nautiques de la terre. A l'heure actuelle, la zone de menace s'étend à l'Est de la Somalie jusqu'à 1500 kilomètres. Les pirates ont recours à des bateaux mères, souvent eux-mêmes piratés et donc anodins, qui permettent de déployer en haute mer et très loin des côtes plusieurs petites embarcations légères aptes à prendre le contrôle d'un navire de grande taille en quelques minutes. Ils s'attaquent de préférence à des navires vulnérables, lents et sans protection qu'ils abordent avec des échelles et des grappins. Si les pirates n'hésitent pas à tirer sur les bâtiments pour les contraindre à stopper, ils ne commettent en général pas d'acte de violence à l'encontre de l'équipage, qui constitue une monnaie d'échange de grande valeur au même titre que la cargaison.

13 La récupération de rançons en échange de leurs prises constitue une activité particulièrement rentable pour de simples pêcheurs dont les revenus sont soudainement devenus confortables. Le versement des rançons est utilement mis à profit pour moderniser l'arsenal des pirates (armement, moyens nautiques, instruments de navigation et de communication) et améliorer leur train de vie ainsi 
que celui de leurs proches. Ils retiennent parfois pendant des semaines bateaux et équipages, le temps moyen de libération d'un navire avoisinant les soixante jours ${ }^{3}$.

\section{Un enjeu international}

L'étendue de la zone de danger piraterie, le grand nombre de navires transitant par le golfe d'Aden et l'extrême rapidité des attaques des pirates rendent pratiquement irréalisable une sécurisation complète de la zone géographique d'action des pirates, tant ce mode d'action nécessiterait des moyens considérables. La solution la plus efficace pour protéger les navires marchands consiste donc à les accompagner par des navires militaires, complétée de préférence par la mise en place à bord d'une équipe de protection embarquée (EPE) composée de commandos marine et par la prise par les armateurs de mesures de protection passives rendant plus difficile l'accès des pirates à bord, en particulier à l'intérieur des navires.

\section{Photo 2 - La protection des navires civils lors d'un accompagnement}

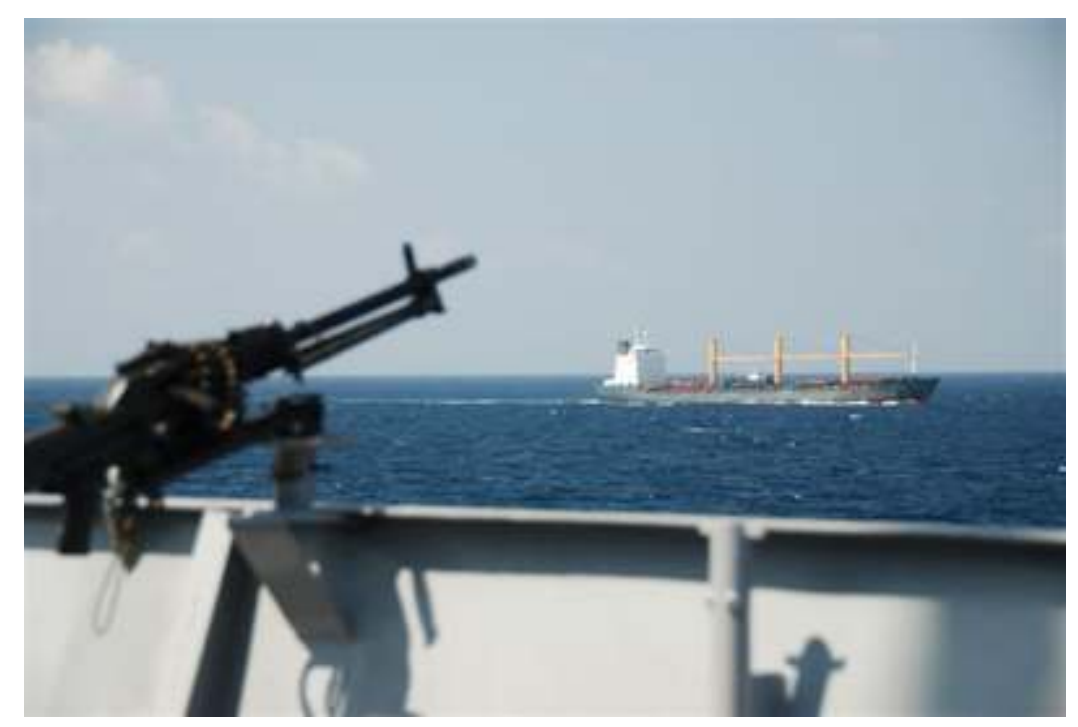

Cliché : auteur.

De telles mesures nécessitent toutefois un grand nombre de moyens militaires: en premier lieu des navires de guerre avec des hélicoptères et des commandos marine embarqués, mais aussi des avions de patrouille et de surveillance maritime pour assurer la surveillance des zones d'action des pirates, des hélicoptères à terre pour assurer le transport des commandos à bord des navires de guerre, sans oublier les états-majors et les systèmes de commandement, de gestion de l'information et de communication par satellite indispensables au commandement des opérations.

Pour faire face à cet enjeu particulier de défense et de sécurité, la France a dû s'investir massivement sur le théâtre. La présence permanente d'ALINDIEN et de son état-major 
embarqué au cœur de cette zone stratégique majeure a permis à la France de jouer un rôle majeur dans la mobilisation de la communauté internationale et dans le processus de mise en place de la première force maritime européenne de l'histoire.

La communauté internationale s'est donc mobilisée en cohérence avec les résolutions du Conseil de sécurité des Nations Unies ${ }^{4}$. Les moyens déployés sur zone se renforcent de semaine en semaine: outre la force Atalanta de l'Union Européenne ${ }^{5}$, première coalition internationale ayant un mandat spécifique de lutte contre la piraterie, et le déploiement, sous pavillon national, de bâtiments de nombreux pays ${ }^{6}$, on a assisté courant janvier à la création, sur proposition des Etats-Unis, de la Task Force 151 au sein d'une coalition de circonstance de plusieurs pays décidés à lutter ensemble contre la piraterie. Initialement constituée d'unités américaines, la TF 151, à laquelle participent occasionnellement les Britanniques, s'est enrichie dernièrement d'une frégate turque et d'unités singapourienne et coréenne. L'OTAN a également envoyé des moyens dans le cadre de la force multinationale maritime SNMG1. En moyenne, ce ne sont pas moins de 25 à 30 unités qui évoluent dans le Golfe d'Aden et au large de la Corne d'Afrique : une dizaine sous Atalanta, 5 en TF151, 4 sous la bannière de l'OTAN et 7 en national tasking.

\section{Le rôle et les actions de la France dans la zone}

Dans son rôle d'acteur des relations internationales, la France entend défendre la liberté des mers et la sécurité des flux maritimes. ALINDIEN s'est ainsi vu confier récemment des prérogatives en terme d'action de l'Etat en mer. Ces responsabilités permettent à notre pays de se donner les moyens juridiques et les outils pour réprimer les infractions dans des zones maritimes hors juridiction française : piraterie, trafic de stupéfiants, immigration illicite. Cette responsabilité civile confiée à un militaire exerçant un commandement opérationnel, à l'instar des responsabilités confiées aux préfets maritimes en métropole, est l'illustration de l'approche de défense et de sécurité souhaitée par le président de la République.

Parmi les missions confiées à ALINDIEN figurent la surveillance et la protection des lignes de communication maritime en océan Indien et en particulier des navires marchands français. A cette fin, il anime depuis 2001 le contrôle naval volontaire en océan Indien (CNVOI). Le CNVOI est un protocole de coopération et d'échange d'informations entre la marine nationale et les armateurs français. Dispositif ancien réactivé en 2001, il permet à tous les navires français dont les armateurs sont partie prenante au CNVOI de bénéficier d'informations et de conseils de prévention. Ces derniers signalent leur itinéraire et leur position, permettant ainsi une intervention rapide en cas de difficultés. Le contrôle naval volontaire s'étend sur toutes les zones de l'océan Indien identifiées "à risque », depuis le canal de Suez jusqu'en mer de Chine méridionale en passant par le golfe d'Aden. Les bâtiments de la marine nationale qui patrouillent dans la zone actualisent en temps réel les conseils donnés et prennent contact systématiquement avec les navires de commerce français rencontrés à la mer.

21 Avec ALINDIEN, ses moyens et ses bases de soutien, la France possède ainsi dans cette région sensible un dispositif interarmées qui confère aux plus hautes autorités politiques et militaires de l'Etat une autonomie d'appréciation de situation, ainsi que les capacités d'anticipation indispensables à la protection des intérêts français. Ce dispositif pré-positionné permet également d'agir rapidement en cas de crise. 
Il en a été ainsi à deux reprises en 2008 et également à une occasion en 2009, à la suite du détournement de trois navires français. ALINDIEN, avec en particulier un renfort de forces spéciales, a alors été amené à conduire trois opérations de libération des otages du voilier de croisière Le Ponant au mois d'avril 2008, du voilier de plaisance Carré d'As en septembre de la même année et du Tanit en avril 2009. Les quinze pirates capturés lors des trois opérations ont été transférés en France pour répondre de leurs actes devant la justice française.

Photo 3 - Interception des pirates par l'EPE d'un bâtiment de la marine nationale

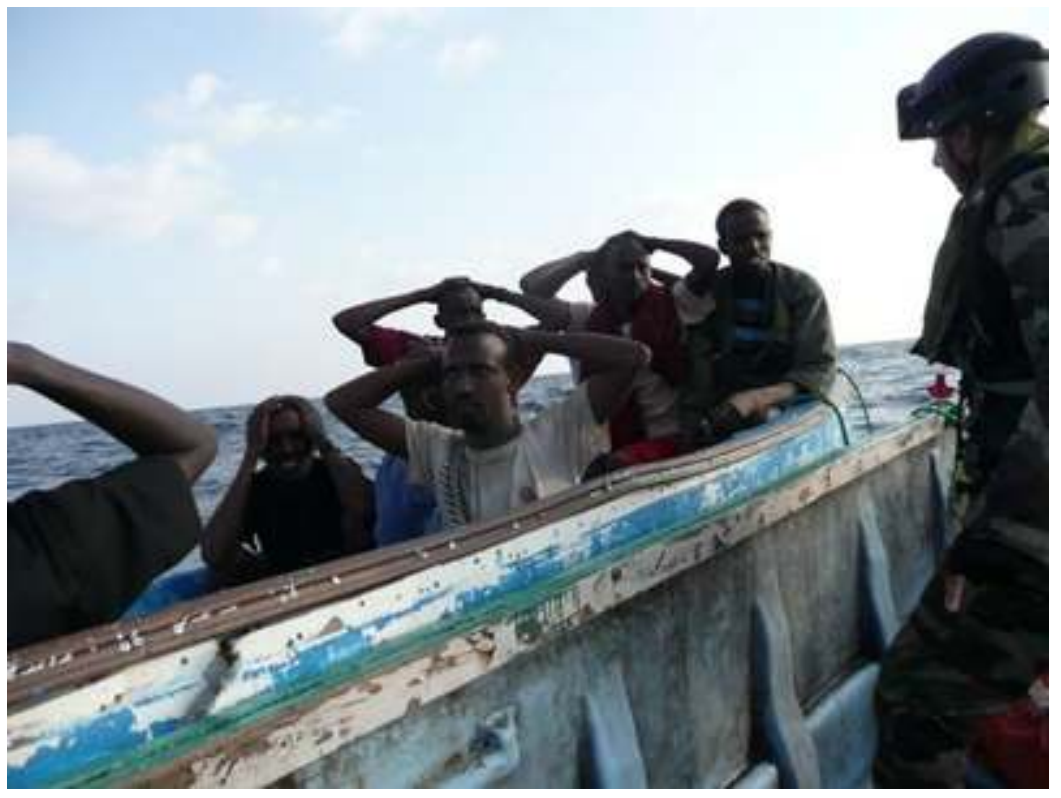

Cliché : auteur.

D'autres actions de lutte contre la piraterie ont été menées par des moyens sous le contrôle opérationnel d'ALINDIEN. Le jeudi 23 octobre 2008, neuf pirates somaliens, interceptés par l'aviso Commandant Birot dans le golfe d'Aden à une centaine de milles nautiques des côtes, ont été remis aux autorités somaliennes. De l'armement individuel et antichar ainsi que du matériel d'abordage ont été découverts dans leurs embarcations à la suite d'une attaque contre un navire marchand. L'année 2009 a vu le nombre d'attaques se poursuivre à un rythme effréné, mais avec une efficacité moindre en termes de prises, en raison de la forte présence internationale. Mais, progressivement, l'action de la France contre la piraterie s'est inscrite dans le cadre de l'Union européenne au fur et à mesure de la montée en puissance de celle-ci dans cette lutte.

\section{L'intervention européenne}

Parmi les premiers pays à réagir à cette situation inquiétante, la France, de concert avec l'Espagne, a milité pour le lancement d'une initiative européenne. Elle a notamment proposé d'assurer l'accompagnement de navires vulnérables dans le golfe d'Aden et poussé à la création d'une cellule de coordination des actions de lutte contre la piraterie à l'échelle européenne : la cellule EU/NAVCO. Cette dernière, instaurée par le Conseil de l'Union européenne le 15 septembre dernier, a joué un rôle d'interface 
entre les armateurs européens et les Etats-membres, disposant de moyens maritimes militaires dans le golfe d'Aden. Ainsi, au cours de cette période intermédiaire, de miseptembre à début décembre 2008, les bâtiments d'ALINDIEN ont assuré une quinzaine d'accompagnements de navires vulnérables, mettant en place à bord, chaque fois que possible, une équipe de protection embarquée. Cinq bâtiments de la marine nationale ont été mobilisés dans cette activité7.

Par la suite, le 15 septembre 2008, le conseil des affaires étrangères de l'Union européenne a décidé le lancement de l'opération Atalanta de lutte contre la piraterie maritime au large des côtes somaliennes. Placée sous commandement opérationnel britannique et sous contrôle opérationnel grec, il s'agit de la première opération navale de la PESD (Politique européenne de sécurité et de défense). Depuis le 8 décembre 2008, cette force agit en appui des résolutions 1814, 1816, 1838 et 1846 et 1851 du conseil de sécurité de l'ONU. Elle escorte les navires du Programme alimentaire mondial, qui acheminent l'aide alimentaire aux populations déplacées de Somalie, et assure la protection des navires vulnérables navigant au large des côtes somaliennes en organisant des convois protégés et des patrouilles destinées à dissuader, prévenir et réprimer les actes de piraterie au large de ces côtes.

26 Au total, une dizaine de pays participent à l'opération Atalanta. Le format de la force navale européenne évolue en permanence et compte en moyenne huit à douze bâtiments du type frégate avec des hélicoptères, et de deux à quatre avions de patrouille maritime.

27 Le contrôle politique de cette force est exercé par le Conseil européen, via le COPS (comité politique et de sécurité), qui en assure également la direction stratégique. Le CMUE (comité militaire de l'Union européenne) assure de son côté le suivi de la bonne exécution de l'opération, conduite sous la responsabilité d'un état-major opératif basé à Northwood, au Royaume-Uni. Ce dernier est armé par 80 personnes, dont six Français.

Grâce à la ratification par l'Union européenne de la convention des Nations-Unies sur le droit de la mer signée en 1982 à Montego Bay et aux résolutions du Conseil de Sécurité des Nations Unies sur lesquelles elle s'appuie, la force dispose du cadre juridique l'autorisant à intervenir en cas d'attaque ou de suspicion d'attaque contre un navire, en haute mer comme dans les eaux territoriales somaliennes. Par ailleurs, elle met en place des accords et des procédures facilitant l'embarquement des équipes de protection embarquées sur les bâtiments marchands et le transfert à terre pour y être jugés des pirates appréhendés en mer.

29 La France a décidé de participer de façon continue à cette opération, ordonnée pour une durée initiale d'un an, en y affectant en permanence une frégate et un avion de patrouille maritime Atlantique 2, basé à Djibouti. Notre pays propose également un soutien logistique en permettant aux nations impliquées de s'appuyer sur les infrastructures aéroportuaires de la base française à Djibouti.

30 Les actions de la force navale européenne (EUNAVFOR) sont coordonnées avec celles de la TF 151, les deux forces étant complémentaires. De façon générale, l'EUNAVFOR coordonne ses activités avec l'ensemble des marines présentes sur la zone.

31 Désormais sous Atalanta, les forces françaises ont continué à avoir des résultats significatifs. Les 2 et 6 janvier 2009, 27 pirates ont ainsi été remis aux autorités somaliennes au large de Bossaso, après avoir été interceptés par l'aviso Premier-maître Lher et la frégate Jean de Vienne alors qu'ils tentaient d'aborder des cargos évoluant dans 
le golfe d'Aden. Les autorités locales se sont engagées à poursuivre les pirates et ont donné au gouvernement français les garanties d'un traitement de ces prisonniers conforme à la dignité humaine et excluant tout recours à la peine de mort. Au mois de janvier, le Jean de Vienne a aussi arrêté 19 pirates et le Floréal 9, tous remis aux autorités puntlandaises. En avril, également dans le cadre de l'opération Atalanta, le Nivôse a procédé dans un premier temps à l'arrestation de 11 pirates remis au Kenya pour y être jugés, puis à celle de trois autres individus remis quant à eux aux Seychelles. Au total, la France a procédé à l'arrestation de 85 pirates. Tous comparaitront devant la justice pour répondre de leurs actes, soit devant une cour française, soit devant des juges somaliens, soit devant la magistrature des pays ayant accepté de juger les pirates sur leur sol : Kenya et Seychelles.

Photo 4 - La passerelle supérieure d'un bâtiment de la marine lors d'un accompagnement toujours en veille

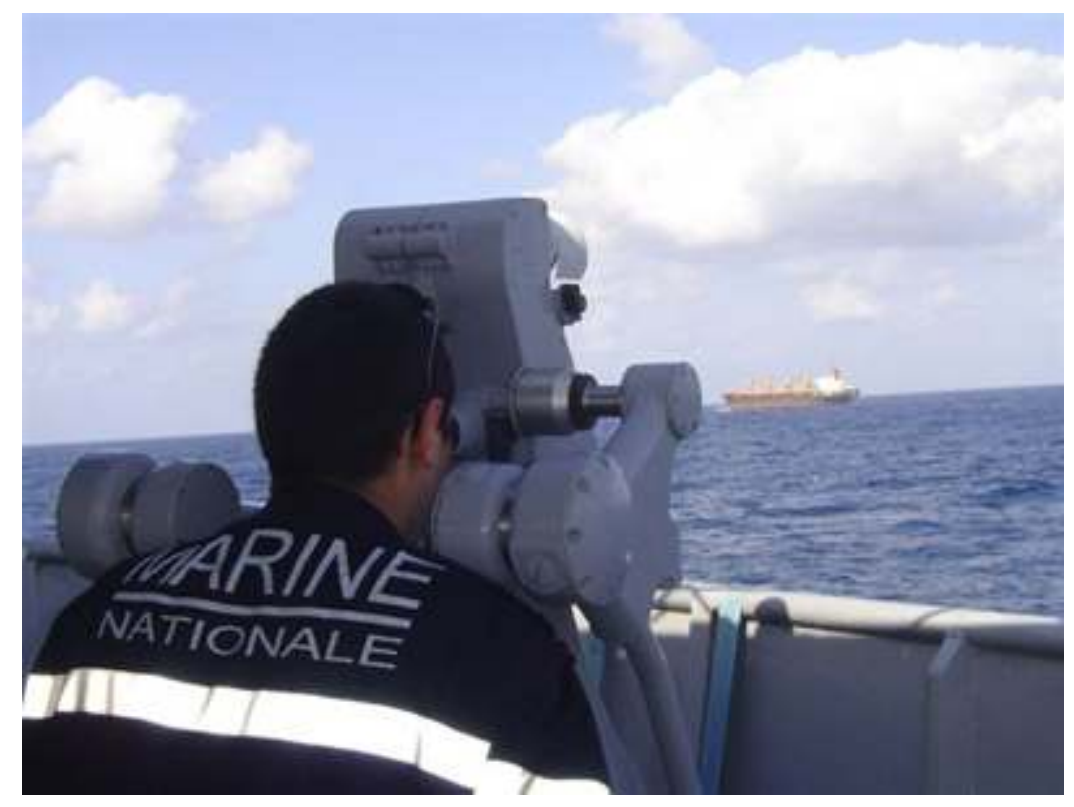

Cliché : auteur.

\section{La nécessité d'une approche globale}

Le grand avantage de l'opération Atalanta réside dans son insertion dans une approche globale conduite par l'Union européenne, union politique dont le champ de compétences s'étend bien au-delà de celui d'une simple coalition militaire. En effet, sous un cadre institutionnel unique, l'Union européenne coordonne l'action militaire d'Atalanta avec son action diplomatique de soutien au processus de Djibouti, destiné à faciliter la mise en place d'un gouvernement stable en Somalie, et avec l'aide économique de la Commission européenne à la Somalie et aux autres Etats riverains du golfe d'Aden.

Cette approche globale doit être privilégiée. En effet, l'instabilité politique et sociale de la Somalie ainsi que les problèmes de pauvreté et de malnutrition qui l'affectent sont autant de facteurs encourageant le recours à la piraterie et à l'argent facilement gagné. Parallèlement à l'opération Atalanta, l'Union européenne s'est donc engagée aux côtés 
du gouvernement fédéral de transition somalien pour trouver des axes de résolution durable de la crise. L'Union européenne soutient ainsi les mesures qui visent à améliorer la situation sécuritaire en Somalie, notamment en apportant une aide financière à l'opération de l'Union africaine en Somalie (AMISOM) et en soutenant la formation d'une force de police somalienne.

Pour la période 2008 à 2013, l'UE s'est également engagée à fournir une allocation de près de 216 millions d'euros, qui sera investie dans les secteurs de la gouvernance, de l'éducation et du développement rural.

Le lancement fin 2007, à la demande du président de la République française, de la mission Alcyon d'accompagnement des navires du Programme alimentaire mondial au large de la Somalie participait déjà à cette logique d'approche globale : du 16 novembre 2007 au 2 février 2008, les avisos CDT Ducuing et PM L'Her ont ainsi permis l'acheminement de près de 30000 tonnes de vivres lors de six rotations entre Mombasa et la Somalie. Cette initiative a enclenché un processus d'accompagnement qui perdure encore aujourd'hui et qui garantit l'approvisionnement d'une population somalienne dont $40 \%$ dépend directement de l'aide alimentaire humanitaire.

Selon le bureau du PAM de Nairobi, depuis le début de la mission Atalanta, 29 navires ont été escortés, ce qui correspond à près de 240000 tonnes de nourriture, soit plus d'1,1 millions de personnes nourries par an.

Du point de vue des accompagnements, $98 \%$ des navires marchands ayant demandé la protection d'Atalanta et ayant respecté les consignes délivrées par la force navale européenne ont transité dans le Golfe d'Aden sans avoir été saisis par des pirates. Selon l'OHQ de Northwood (chiffres à la mi-juin), plus de 6200 navires marchands ont été enregistrés sur le site MSCHOA depuis le $1^{\text {er }}$ janvier 2009.

Ces diverses actions témoignent de la détermination française et européenne à lutter efficacement contre la piraterie, dans le respect des réglementations nationales et internationales en vigueur, ainsi que de la souveraineté des nations concernées. Un engagement qui a su entraîner une mobilisation importante de la communauté internationale dont le dispositif global, toutes forces confondues, a fait chuter, ces derniers mois, la proportion des navires détournés par rapport au nombre d'attaques. Un répit relatif qui ne peut s'inscrire dans la durée qu'en menant, en parallèle de l'action militaire et dans une approche globale, une politique d'aide à la Somalie afin d'œuvrer à la réintégration de ce pays dans le giron des nations, avec des forces de police et de sécurité aptes à lutter contre les gangs mafieux qui sévissent sur son territoire.

Engagée initialement sur un mandat d'une année, la force Atalanta vient d'ailleurs de voir son mandat prolongé pour une année supplémentaire, jusqu'à fin 2010. Soulignant "qu'Atalanta a démontré sa capacité d'agir efficacement contre la piraterie » et que celle-ci «constituera vraisemblablement une menace au-delà de l'échéance», les ministres des Affaires étrangères des 27 ont en effet convenu de poursuivre leur effort.

La soudaine augmentation des actes de piraterie au large de la corne de l'Afrique a permis de démontrer la pertinence du dispositif des armées françaises en océan Indien. Forte de son expérience, de ses succès dans les affaires de prises d'otages, de sa doctrine d'usage proportionné de la force et de son attachement à la légalité internationale aussi bien que nationale, la France est considérée comme une référence en matière de lutte contre la piraterie. ALINDIEN, bras armé du chef d'Etat-major des 
armées sur cette zone, est aussi le trait d'union essentiel entre les commandements français et étrangers, interalliés ou de coalition, présents sur le théâtre. La France a en particulier été un acteur majeur de la montée en puissance de l'Union européenne dans la lutte contre la piraterie, Union européenne qui est certainement l'organisation internationale la mieux placée pour lutter contre la piraterie grâce à la capacité de coordination que lui confère l'approche globale accessible uniquement à une union politique qui ne se limite pas aux aspects militaires.

\section{NOTES}

1. Mallet, J.-Cl. (dir.), Juin 2008. Livre blanc sur la Défense et la Sécurité nationale (volume 1). Paris, Odile Jacob - La Documentation française, 350 p.

2. En particulier dans le domaine naval : groupe aéronaval, groupes amphibie ou de guerre des mines.

3. A signaler toutefois le cas du remorqueur Yenegoa Ocean (pavillon Panama, équipage nigérian), qui était aux mains des pirates depuis fin juillet 2008 et qui n'a été libéré contre paiement d'une rançon qu'après 304 jours de captivité.

4. Résolutions 1814 (15/5/2008), 1816 (2/6/2008), 1838 (7/10/2008), 1846 (2/12/2008) et 1851 (16/12/2008).

5. La force Atalanta compte à ce jour des unités françaises, espagnoles, allemandes, grecques, italiennes et suédoises.

6. Pays déployant des bâtiments opérant indépendamment : Russie, Chine, Inde, Malaisie, Arabie Saoudite, Iran et Japon.

7. L'aviso Commandant Birot, le bâtiment de commandement et de ravitaillement Var et les frégates Courbet, Nivôse et Jean de Vienne

\section{RÉSUMÉS}

La piraterie dans le Golfe d'Aden et au large des côtes somaliennes connaît depuis 2008 un pic important. Précurseur dans la lutte contre ce fléau, organisant des accompagnements de navires vulnérables, conduisant des assauts pour libérer des otages, œuvrant pour le vote de résolutions à l'ONU, la France a été à la pointe de la mobilisation internationale qui s'est progressivement organisée. Ses moyens opèrent désormais sous bannière européenne au sein de la force mise sur pied dans le cadre de l'opération Atalanta. Mais d'autres forces se sont également constituées avec notamment la Task Force 151, emmenée par les Américains, et l'OTAN ainsi que de nombreux pays agissant indépendamment. Si les attaques se poursuivent, elles connaissent un taux d'efficacité moindre. Seule une approche globale de la problématique somalienne permettra à long terme de sécuriser cet axe maritime stratégique qu'est le Golfe d'Aden pour le commerce mondial. A ce jour, seule l'Union européenne semble s'engager dans cette voie. 
Since 2008, piracy in the Gulf of Aden and off Somalia has considerably increased. France took initiatives and mobilized the international community by escorting vulnerable vessels, by freeing hostages and by working for resolutions at the United Nations. The French means are now used under the European flag, within Atalanta Operation. In parallel, some other operations have been set up by the USA (Task Force 151), NATO and various other States. Piracy goes on but the success rate is getting lower and lower. At the end of the day, only a global approach of the Somalian issues will be able to secure the Gulf of Aden, which is a strategic Sea Line Of Communication for the world trade. Today, only the European Union seems to follow this method.

\section{INDEX}

Mots-clés : ALINDIEN, Atalanta, golfe d'Aden, piraterie, Somalie Palabras claves : ALINDIEN, Atalanta, Gulf of Aden, piracy, Somalia

Thèmes : Sur le Champ - Sur le Terrain

\section{AUTEUR}

\section{VICE-AMIRAL GÉRARD VALIN}

Le vice-Amiral Gérard Valin (alindien@free.fr) est commandant la zone maritime de l'océan Indien. 